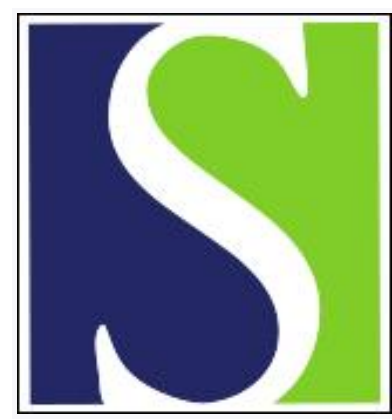

Scand J Work Environ Health 2012;38(1):19-26

https://doi.org/10.5271/sjweh.3200

Published online: 10 Oct 2011, Issue date: Jan 2012

Occupational noise exposure, social class, and risk of ischemic heart disease and all-cause mortality - a 16-year follow-up in the Copenhagen Male Study

by Suadicani P, Hein HO, Gyntelberg F

Affiliation: Copenhagen Male Study, Epidemiologic Research Unit (PS, $\mathrm{HOH}, \mathrm{FG}$ ), Copenhagen University Hospital, Bispebjerg, Denmark. PSUA0001@bbh.regionh.dk

Refers to the following text of the Journal: 2005;31(4):291-299

The following articles refer to this text: 2012;38(1):1-3;

2013;39(1):106-111

Key terms: all-cause mortality; cardiovascular disease; Copenhagen Male Study; CVD; epidemiology; ischemic heart disease; mortality; noise; noise exposure; occupational noise

This article in PubMed: www.ncbi.nlm.nih.gov/pubmed/21986898

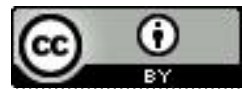




\title{
Occupational noise exposure, social class, and risk of ischemic heart disease and all-cause mortality - a 16-year follow-up in the Copenhagen Male Study by Poul Suadicani, DDS, DMSc, ${ }^{1}$ Hans Ole Hein, MD, ${ }^{1,2}$ Finn Gyntelberg, MD, DMSc ${ }^{1}$
}

\begin{abstract}
Suadicani P, Hein HO, Gyntelberg F. Occupational noise exposure, social class, and risk of ischemic heart disease and all-cause mortality - a 16-year follow-up in the Copenhagen Male Study. Scand J Work Environ Health. 2012;38(1):19-26. doi:10.5271/sjweh.3200
\end{abstract}

\begin{abstract}
Objective Long-term exposure to occupational noise may be associated with an increased risk of ischemic heart disease (IHD) and all-cause mortality. However, the issue remains unsettled. Only a small number of longitudinal studies have been carried out, and control for potential confounders including a strong correlate of noise exposure namely social class may have been insufficient.
\end{abstract}

Methods We carried out a 16-year follow-up of 2998 men aged 53-75 years without overt cardiovascular disease.

Result Overall, 197 men (6.6\%) died due to IHD and 1192 (39.8\%) from all-causes. Of the 2998 men, 1008 $(33.6 \%)$ reported exposure to occupational noise for $\geq 5$ years [mean 25.4, standard deviation (SD) 12.5 years]; among these men, $47.3 \%$ reported hearing impairment versus only $24.8 \%$ among unexposed men $(63.0 \%)$. Referencing unexposed men, the hazard ratio (HR) for IHD mortality was 0.97 [95\% confidence interval $(95 \%$ CI) $0.71-1.33$ ], and the HR for all-cause mortality was 1.01 (95\% CI $0.89-1.15)$ when adjusting for potential confounders: age, hearing impairment, blood pressure, diabetes, fasting serum triglycerides and high-density lipoprotein cholesterol, low-density lipoprotein cholesterol, glucosuria, cancer, body mass index, alcohol, tobacco, leisure-time physical activity, and social class. Stratified analyses of high and low social classes confirmed the overall results.

Conclusion Cumulative occupational exposure to noise was strongly associated with hearing impairment, but not with death from either IHD or all-cause mortality in a long-term follow-up adjusting for established cardiovascular risk factors including low social class.

Key terms cardiovascular disease; CVD; epidemiology; mortality.

Occupational noise exposure frequently occurs in the working environment and is a global occupational health hazard with considerable social and physiological impact. Hearing loss is the most well-established health effect. In the European Union, a survey among workers from 2000 , revealed that $28 \%$ were occupationally exposed to noise one fourth of the time at a level of approximately $85-90 \mathrm{~dB}(1)$.

Other suspected health effects from long-term excessive occupational noise exposure include elevated blood pressure and increased risk of ischemic heart disease (IHD). However, these latter health effects are both subject to controversy and thus remain unsettled
(2-4). In an 8-year incidence study on cardiovascular disease (CVD) and all-cause mortality, the Cardiovascular Occupational Risk Factors Determination in Israel (CORDIS) study found no statistically significant increased risk of CVD mortality among subjects exposed to noise levels $>85 \mathrm{~dB}$ for $>10$ years (2). Statistically significant higher all-cause mortality was found in this group. Of 2733 persons, 95 died from any cause, 14 among the 273 noise-exposed, hazard ratio (HR) 1.97 [95\% confidence interval $(95 \% \mathrm{CI})$ 1.01-3.83] adjusted for age, smoking, hearing protector use, and leisure-time physical activity. No adjustment was made for social class.

1 Copenhagen Male Study, Epidemiologic Research Unit (PS, HOH, FG), Copenhagen University Hospital, Bispebjerg, Denmark.

2 Research Centre for Prevention and Health ( $\mathrm{HOH})$, Glostrup University Hospital, Denmark. 
In particular, as stated by McNamee et al (4), apart from their own study, only a few other prospective epidemiological studies have addressed the issue of occupational noise exposure as a risk factor for IHD $(2,5,6)$. McNamee et al (4) concluded that, compared to unexposed men, no statistically robust risk could be found for an increased risk of IHD among nuclear power workers exposed to noise after adjustment for blood pressure, height, body mass index (BMI), and smoking. In a register cohort study of 27464 blue-collar workers from lumber mills in British Columbia, Davies et al (5) found a relative risk of 1.5 (95\% CI 1.1-2.2) for circulatory deaths $(\mathrm{N}=2510)$ in the highest noise-exposed group; smoking did not explain the association. In an 18-year follow-up study of 6005 middle-aged industrially employed men, Virkkunen et al (6) found a relative risk of 1.54 (95\% CI 1.28-1.86) for those exposed to continuous or impulse noise when adjusting for classical risk factors for coronary heart disease. For blue-collar workers, the relative risk was 1.29 (95\% CI 1.05-1.57).

Several methodological challenges confront researchers attempting to find out whether a causal association between occupational exposure to noise and risk of IHD may exist or not. Most important among these are (i) temporality: the exposure must precede the outcome; (ii) validity of the exposure assessment and validity of the outcome(s); and (iii) sufficient confounder control to minimize residual confounding.

In the Copenhagen Male Study, it is possible to take into account most of these methodological challenges. Accordingly, in a 16-year follow-up we decided to test the hypothesis that an association exists between cumulative lifetime exposure to occupational noise and risk of IHD and all-cause mortality.

\section{Methods}

The Copenhagen Male Study was set up in 1970 as a prospective cardiovascular study of Caucasian middleaged men employed at 14 large workplaces in Copenhagen $(7,8)$. Overall, 6125 men were invited to participate; 5249 men $(87 \%)$ agreed.

In 1985-1986 a new baseline was established that was used for the analyses in the present study together with information on blood pressure measured in 1970 1971. All survivors from the 1970 study were traced through the Danish National Civil Registry. Between June 1985 and June 1986, 4505 men except 34 emigrants, were invited to take part in this new study; 3387 men $(75 \%)$ agreed to participate and gave informed consent. Their mean age was 63 years, range 53-75 years. We excluded 33 invalid questionnaires, leaving 3354 men for the analysis; within this group 319 men were excluded because of CVD (angina pectoris, acute myocardial infarction, stroke, and/or intermittent claudication); in all, 2998 men with useful information on occupational noise exposure were included.

\section{Baseline measurements}

All men were interviewed by a physician about a previously completed questionnaire and were examined with measurements of height, body weight, and blood pressure; a fasting venous blood sample for the determination of serum lipids and a urine sample for determination of glucosuria were taken.

Information on a number of physical and chemical occupational exposures was obtained from the questionnaire, including information on noise exposure. Longterm exposure was defined as frequent occupational exposure for $\geq 5$ years. The question was phrased as follows. "Have you at your present or previous workplaces often (ie, several times/week or more) been exposed to noise at a level where it is necessary to raise your voice?" Answer options were "no" and "yes", and "if yes, for how many years?"

Total weekly use of alcohol was calculated from questionnaire items about average use on weekdays and weekends. Intakes of beer, wine, and spirits were reported separately. One drink corresponded to 10-12 g of ethanol. The men reported whether they had noninsulin dependent diabetes mellitus (ie, type 2 diabetes).

Leisure-time physical activity was assessed with the question: "Which description most precisely covers your pattern of leisure-time physical activity? (i) You are almost entirely sedentary or perform light leisuretime physical activity less than two hours a week; (ii) You perform light leisure-time physical activity two to four hours a week; (iii) You perform light leisure-time physical activity more than four hours a week or more vigorous activity two to four hours a week; (iv) You perform highly vigorous leisure-time physical activity more than four hours a week or regular exercise or competitive sports several times a week." For analytical purposes, groups 3 and 4 were pooled and are referred to as the "high activity" group.

The men were subdivided into five social classes, using a modification of a system by Svalastoga (9) based on the level of education and the job profile. Men in social class I were predominantly academics and wellskilled administrators or executives; men in social class V were unskilled and semi-skilled workers.

The men classified themselves as never having smoked, being a previous smoker, or being a current smoker. Current tobacco smoking was calculated from information about the number of cigarettes, cheroots, cigars, or the weight of pipe tobacco smoked daily. One cigarette was taken as equivalent to $1 \mathrm{~g}$ of tobacco, one 
cheroot as $3 \mathrm{~g}$, and one cigar as $4 \mathrm{~g}$. As previously estimated by means of measurements of serum cotinine, the validity of tobacco reporting was high (10). Cumulative tobacco consumption (in pack-years) was calculated on the basis of information about mean total daily use of tobacco in grams multiplied by the time as a smoker in years divided by 20 . In the multivariable analyses, cumulative tobacco consumption was included as a predictor using quintile categories; the cut-off points were $3.3,18.0,31.5$ and 45.0 pack-years, respectively.

BMI was calculated as weight in $\mathrm{kg}$ divided by height in $\mathrm{m}^{2}$.

Serum concentrations of total cholesterol, high-density lipoprotein cholesterol (HDL-C) and triglycerides were determined using enzymatic assays (BoehringerMannheim Biochemica, Mannheim, Germany) (11-14). Fasting lipids were measured only once for each subject. Definitions of high-serum triglycerides $(\geq 1.70 \mathrm{mmol} / \mathrm{L})$, low HDL-C $(\leq 1.03 \mathrm{mmol} / \mathrm{L})$, high systolic blood pressure $(>130 \mathrm{~mm} \mathrm{Hg})$, and high diastolic blood pressure $(>85 \mathrm{~mm} \mathrm{Hg}$ ), were based on criteria for definition of the metabolic syndrome according to the Adult Treatment Panel III (15). Low-density lipoprotein cholesterol (LDL-C) was calculated using the indirect method as developed by Friedewald (16). Urine samples were examined for glucosuria using a glucose kinase method (Clinistix ${ }^{\circledR}$, Bayer AG, Leverkusen, Germany).

Blood pressure was measured on the right arm with the subject seated and after $\geq 10$ minutes of rest, using a manometer developed by the London School of Hygiene and Tropical Medicine (17). Information on hypertension treatment was obtained from the questionnaire. For the analyses, high blood pressure was defined as receiving treatment, having a systolic blood pressure $>130 \mathrm{~mm} \mathrm{Hg}$, or having a diastolic blood pressure $>85 \mathrm{~mm} \mathrm{Hg}$.

All men who had taken part in the 1985-1986 examination were traced from registers. A history of cancer was obtained from the Cancer Registry covering all cases of Denmark from 1943 onwards. For the incidence study, information was obtained from death certificate diagnoses within the follow-up period 1985-1986 to 31 December 2001. We used diagnoses from official national registers; IHD diagnoses were codes 410-412 from the International Classification of Diseases (ICD), $8^{\text {th }}$ revision, and, from 1994, codes I20-I25 from ICD, $10^{\text {th }}$ revision.

\section{Statistical analysis}

Basic statistical analyses, including trend tests (Kendall's $\tau$ B and test for linearity in analysis of variance, Chi-squared analysis and test for deviation in analysis of variance, and regression analyses) were performed. Relative risks were estimated by $\exp (B)$, where $B$ is the hazard coefficient for the variable of interest in Cox's proportional hazards regression model, using forced entry models and different adjustment criteria (18). Assumptions regarding the use of Cox's proportional hazards were met by inspection of the $\log$ minus $\log$ function at the covariate mean. A two-sided probability value of $\mathrm{P}<0.05$ was a priori taken as significant.

\section{Results}

\section{Baseline characteristics}

Table 1 shows baseline characteristics of men in three groups according to occupational noise exposure: the unexposed group consisting of $63 \%$ of the men, a small group of $3.3 \%$ with a short-term exposure history of 1-4 years of relevant occupational exposure to noise, and a fairly large group (33.6\% of the men) with $\geq 5$ years of occupational exposure to noise. The mean exposure time in this group was high (24.5 years), providing a strong exposure contrast between those exposed and the unexposed group. Due to sample size alone, the results associated with the short-term exposed group should be interpreted conservatively, in particular with respect to the incidence results presented in tables 2,3 , and 4 . With respect to lifestyle, differences between the three groups were small. There were no differences in smoking prevalence and alcohol consumption, although cumulative smoking calculated as pack years was slightly higher among those with the highest level of noise exposure. Interestingly, systolic as well as diastolic blood pressure was higher among the unexposed men, as was the case for the combined hypertension variable. In contrast, all lipid fractions and BMI were higher among the longterm noise-exposed group. Statistically, the strongest associations were found between the occupational noise exposure group and hearing impairment and social class.

\section{Mortality incidence results}

Table 2 shows risk of IHD mortality and all-cause mortality according to occupational noise exposure group. Referencing the unexposed group, a marginally and not significantly higher risk of $13 \%$ was found for the highest noise-exposure group; differences in lifestyle and social class explained most of this; in the final model including all confounders, the HR was 0.97 (95\% CI 0.71-1.33). A similar pattern was found with respect to all-cause mortality with a weakly and not significantly increased risk of $5 \%$, an increase that completely vanished when taking into account lifestyle and social class differences between those with high noise exposure and those without a history of occupational noise exposure. In the final model including all confounders, the HR was 1.01 (95\% CI 0.89-1.15). 
Table 1. Lifestyle and other characteristics according to self-assessed occupational noise exposure. Values presented are mean and standard deviation (SD) or frequency (Freq) in percent. [BMI=body mass index; BP=blood pressure; HDL-C=high-density lipoprotein cholesterol; LDL-C=low-density lipoprotein cholesterol; LTPA=leisure-time physical activity; TG=triglycerides.]

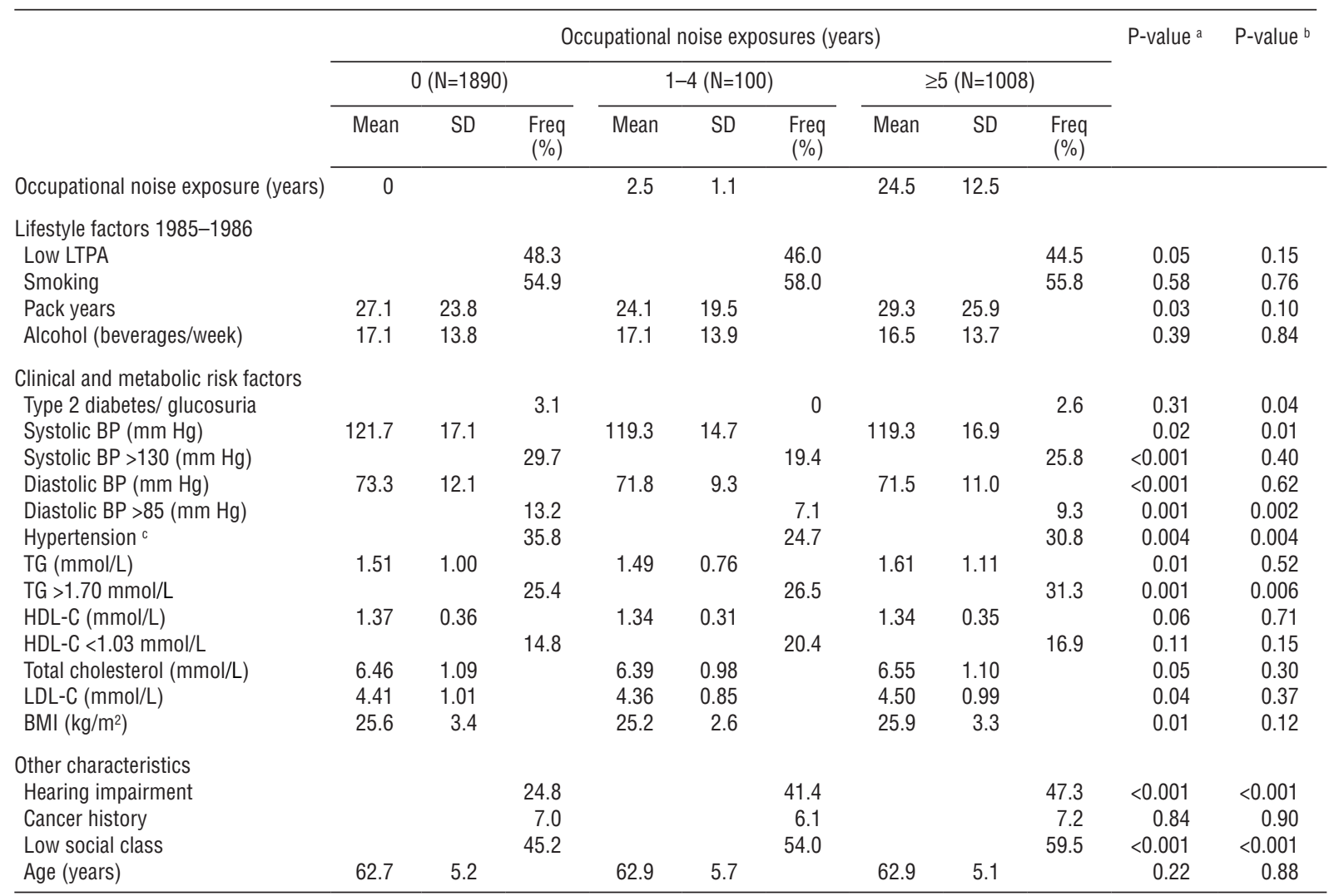

a P-values of trend test (Kendall's tau B) or test for linearity in analysis of variance.

b P-values of Chi-square test or test for deviation in analysis of variance.

c Defined as treatment due to hypertension, systolic blood pressure $>130 \mathrm{~mm} \mathrm{Hg}$, or diastolic blood pressure $>85 \mathrm{~mm} \mathrm{Hg}$.

Table 2. Hazard ratios (HR) for ischemic heart disease (IHD) mortality and all-cause mortality according to occupational noise exposure following different adjustment criteria. Cox proportional hazards regression analyses with forced entry of variables. [95\% $\mathrm{Cl}=95 \%$ confidence interval.]

\begin{tabular}{|c|c|c|c|c|c|c|c|c|}
\hline & \multicolumn{8}{|c|}{ Occupational noise exposure (years) } \\
\hline & \multicolumn{2}{|c|}{$0(N=1890)$} & \multicolumn{3}{|c|}{$1-4(N=100)$} & \multicolumn{3}{|c|}{$\geq 5(\mathrm{~N}=1008)$} \\
\hline & $\%$ a & $\mathrm{HR}$ & $\%$ a & $\mathrm{HR}$ & $95 \% \mathrm{Cl}$ & $\%$ a & $\mathrm{HR}$ & $95 \% \mathrm{Cl}$ \\
\hline IHD mortality & 6.4 & & 4.0 & & & 7.2 & & \\
\hline Age & & ref & & 0.60 & $0.22-1.63$ & & 1.13 & $0.84-1.51$ \\
\hline Age + lifestyle and social class ${ }^{b}$ & & ref & & 0.56 & $0.21-1.53$ & & 1.03 & $0.76-1.39$ \\
\hline Age + clinical factors ${ }^{c}$ & & ref & & 0.67 & $0.25-1.82$ & & 1.06 & $0.78-1.43$ \\
\hline Age + all potential confounders ${ }^{d}$ & & ref & & 0.64 & $0.24-1.75$ & & 0.97 & $0.71-133$ \\
\hline All-cause mortality & 39.1 & & 39.0 & & & 41.3 & & \\
\hline Age & & ref & & 0.98 & $0.71-1.35$ & & 1.05 & $0.93-1.19$ \\
\hline Age + lifestyle and social class ${ }^{b}$ & & ref & & 0.96 & $0.69-1.33$ & & 1.00 & $0.89-1.14$ \\
\hline Age + clinical factors ${ }^{c}$ & & ref & & 1.02 & $0.74-1.42$ & & 1.04 & $0.92-1.18$ \\
\hline Age + all potential confounders ${ }^{d}$ & & ref & & 1.00 & $0.71-1.39$ & & 1.01 & $0.89-1.15$ \\
\hline \multirow{3}{*}{\multicolumn{9}{|c|}{$\begin{array}{l}\text { a Crude incidence. } \\
\text { b LTPA (leisure-time physical activity), cumulative tobacco consumption (cigarette equivalents), alcohol intake, and social class. } \\
\text { c Type } 2 \text { diabetes/glucosuria, hypertension, high-serum triglycerides (TG), low-serum high-density lipoprotein cholesterol (HDL-C), and low-density lipo- } \\
\text { protein cholesterol (LDL-C). }\end{array}$}} \\
\hline & & & & & & & & \\
\hline & & & & & & & & \\
\hline $\begin{array}{l}\text { d LTPA, cumulative tobacco consun } \\
\text { class. }\end{array}$ & & & & & $\mathrm{n}$, high-serun & & & and social \\
\hline
\end{tabular}


Table 3 shows the results of Cox proportional hazards regression analyses for IHD mortality separately for high and low social classes. Overall, the risk of IHD mortality was higher among the lower social classes in all noise exposure groups, but the risk associated with noise exposure was not significant for either the high or low social classes.

Correspondingly, table 4 shows the results of Cox proportional hazards regression analyses for all-cause mortality separately for high and low social classes. Again, the risk of all-cause mortality was higher among the lower social classes in all noise exposure groups, but the risk associated with noise exposure was not significant for either the high or low social classes.

\section{Additional analyses}

In order to maximize the exposure contrast, we compared two groups: (i) men with no hearing impairment and no history of occupational noise exposure $(\mathrm{N}=1422)$ and (ii) men with self-assessed hearing impairment and a history of long-term occupational noise exposure ( $\mathrm{N}=475)$. Referencing men with neither hearing impairment nor noise exposure, the HR for the risk of IHD mortality of men

Table 3. Hazard ratios (HR) for ischemic heart disease mortality according to occupational noise exposure among high and low social classes following different adjustment criteria. Cox proportional hazards regression analyses with forced entry of variables. $[95 \% \mathrm{Cl}=95 \%$ confidence limits.]

\begin{tabular}{|c|c|c|c|c|c|c|c|c|c|c|c|}
\hline & \multicolumn{11}{|c|}{ Occupational noise exposure (years) } \\
\hline & \multicolumn{3}{|c|}{0} & \multicolumn{4}{|c|}{$1-4$} & \multicolumn{4}{|c|}{$\geq 5$} \\
\hline & $\mathrm{N}$ & $\%^{a}$ & $\mathrm{HR}$ & $\mathrm{N}$ & $\%{ }^{a}$ & $\mathrm{HR}$ & $95 \% \mathrm{Cl}$ & $\mathrm{N}$ & $\%^{a}$ & $\mathrm{HR}$ & $95 \% \mathrm{Cl}$ \\
\hline High social classes (I-III) & 1036 & 5.8 & & 46 & 2.2 & & & 408 & 5.6 & & \\
\hline Age & & & ref & & & 0.42 & $0.06-3.00$ & & & 0.95 & $0.59-1.54$ \\
\hline Age + lifestyle and social class ${ }^{b}$ & & & ref & & & 0.40 & $0.05-2.88$ & & & 0.88 & $0.54-1.45$ \\
\hline Age + clinical factors $\mathrm{C}$ & & & ref & & & 0.48 & $0.07-3.46$ & & & 0.78 & $0.47-1.31$ \\
\hline Age + all potential confounders ${ }^{d}$ & & & ref & & & 0.44 & $0.06-3.21$ & & & 0.72 & $0.42-1.22$ \\
\hline Low social classes (IV-V) & 853 & 7.0 & & 54 & 5.6 & & & 599 & 8.4 & & \\
\hline Age & & & ref & & & 0.63 & $0.20-2.01$ & & & 1.14 & $0.78-1.66$ \\
\hline Age + lifestyle and social class ${ }^{b}$ & & & ref & & & 0.68 & $0.21-2.18$ & & & 1.13 & $0.77-1.66$ \\
\hline Age + clinical factors ${ }^{c}$ & & & ref & & & 0.71 & $0.22-2.27$ & & & 1.14 & $0.78-1.69$ \\
\hline Age + all potential confounders ${ }^{d}$ & & & ref & & & 0.77 & $0.24-2.48$ & & & 1.13 & $0.75-1.68$ \\
\hline
\end{tabular}

Table 4. Hazard ratios (HR) for all-cause mortality according to occupational noise exposure among high and low social classes following different adjustment criteria. Cox proportional hazards regression analyses with forced entry of variables. [ $95 \% \mathrm{Cl}=95 \%$ confidence limits.]

\begin{tabular}{|c|c|c|c|c|c|c|c|c|c|c|c|}
\hline & \multicolumn{11}{|c|}{ Occupational noise exposure (years) } \\
\hline & \multicolumn{3}{|c|}{0} & \multicolumn{4}{|c|}{$1-4$} & \multicolumn{4}{|c|}{$\geq 5$} \\
\hline & $\mathrm{N}$ & $\%^{a}$ & $\mathrm{HR}$ & $\mathrm{N}$ & $\%^{a}$ & $\mathrm{HR}$ & $95 \% \mathrm{Cl}$ & $\mathrm{N}$ & $\%^{a}$ & $\mathrm{HR}$ & $95 \% \mathrm{Cl}$ \\
\hline High social classes (I-III) & 1036 & 35.2 & & 46 & 34.8 & & & 408 & 37.2 & & \\
\hline Age & & & ref & & & 1.12 & $0.68-1.84$ & & & 1.04 & $0.86-1.26$ \\
\hline Age + lifestyle and social class ${ }^{b}$ & & & ref & & & 1.09 & $0.66-1.81$ & & & 0.99 & $0.82-1.21$ \\
\hline Age + clinical factors ${ }^{c}$ & & & ref & & & 1.14 & $0.69-1.88$ & & & 1.00 & $0.82-1.22$ \\
\hline Age + all potential confounders ${ }^{d}$ & & & ref & & & 1.11 & $0.67-1.85$ & & & 0.96 & $0.79-1.18$ \\
\hline Low social classes (IV-V) & 853 & 43.7 & & 54 & 42.6 & & & 599 & 44.1 & & \\
\hline Age & & & ref & & & 0.83 & $0.55-1.27$ & & & 0.98 & $0.84-1.15$ \\
\hline Age + lifestyle and social class ${ }^{b}$ & & & ref & & & 0.88 & $0.57-1.35$ & & & 1.00 & $0.85-1.18$ \\
\hline Age + clinical factors ${ }^{c}$ & & & ref & & & 0.89 & $0.58-1.37$ & & & 0.99 & $0.84-1.17$ \\
\hline Age + all potential confounders ${ }^{d}$ & & & ref & & & 0.93 & $0.59-1.45$ & & & 1.03 & $0.87-1.22$ \\
\hline
\end{tabular}


with combined hearing impairment and long-term noise exposure was 1.24 (95\% CI 0.84-1.82) adjusted for age only; adjusting also for social class attenuated the HR to 1.17 (95\% CI $0.79-1.73)$; a corresponding analysis of all-cause mortality gave HR of 1.0 (95\% CI 0.85-1.18) and 0.95 (95\% CI $0.81-1.12)$, respectively. Finally, to ascertain the relevance of the reporting of work-related noise exposure, we investigated the association between self-assessed occupational noise exposure and job titles of study participants at the time of entry into the study in 1970-1971. For men with long occupational noise exposure, by order of occurrence and compared to men with no reported noise exposure, job titles were: (i) engine driver: $12 \%$ and $<1 \%$, respectively; (ii) train guards: $8 \%$ and $<3 \%$; (iii) unskilled workers: $7 \%$ and $<3 \%$; (iv) craftsmen: $7 \%$ and $<2 \%$; (v) smiths: $4 \%$ and $<1 \%$; and (vi) mechanics: $5 \%$ and $<2 \%$.

\section{Discussion}

This study supports the premise that cumulative longterm occupational noise exposure does not increase the risk of premature mortality from either IHD or all causes.

As mentioned in the introduction, the methodological challenges of attempting to suggest causality are temporality, validity of the exposure and the outcome, and sufficient confounder control.

Temporality. We overcame this challenge in our study by using a 16-year period of follow-up. The study was a true incidence study since all men with a history of clinically overt CVD were excluded at baseline.

Validity of the exposure. Occupational noise exposure was solely based on questionnaire information. No objective measurements were available of the actual noise levels to which each study participant had been exposed during his working life. Nor was any objective information obtained on the duration of noise exposure during each participant's working hours. Concerning the validity of noise assessment methods, monitoring cumulative noise exposure objectively on an individual level during work life has never been performed nor will it in all likelihood ever be possible to do so; thus we shall have to use less-accurate exposure measures. The only incidence study where measurements of noise have been used is the study by McNamee et al (4). This exposure assessment was retrospective for each man based on company histories and noise survey records during a 33-year period plus information on the use of hearing aids. This assessment method may probably be the best you can achieve for practical purposes. However, also such an exposure assessment will be less than optimal.

The questionnaire information has previously been validated indirectly as reported in two publications from the Copenhagen Male Study $(19,20)$ based on analysis of the entire 1985-1986 cohort including those with a CVD history. A strong correlation was found between reported years of occupational noise exposure and self-reported hearing impairment. In a subsample of the cohort, Parving et al (20) showed a clear agreement between self-reported hearing impairment and reduced hearing capacity as measured by pure tone audiometry. These results support that, on a group level, self-reported noise exposure has a sufficient validity in the Copenhagen Male Study cohort to be used at least for categorization of men with a very low or very high cumulative exposure to occupational noise. A risk of misclassification may exist if men with hearing impairment had a different risk of IHD than others. We analyzed this possibility, controlling in the analysis for age and social class. Compared to other men, the HR for men with hearing impairment was 1.14 (95\% CI 0.86-1.53), $\mathrm{P}=0.36$ (not shown in table).

Furthermore, the percentage of working men in the Copenhagen Male Study occupationally exposed to a self-reported noise level where it is necessary to raise your voice to be heard $(33.6 \%)$, was quite similar to the frequency reported by Nelson et al, namely, $28 \%$ among European Union workers (1). In this study, almost half of the men with occupational noise exposure for $\geq 5$ years claimed to have a hearing impairment, twice as many as among men who had not been relevantly exposed to noise. In summary, the validity of exposure to occupational noise must be considered sufficient for analysis of noise exposure as a potential risk factor for IHD as well as all-cause mortality.

Validity of the outcomes. In Denmark, every citizen has a unique personal number of identification held in the Central Person Register (CPR), which includes date of birth and four additional numbers. This identification system was introduced in 1969. The CPR number makes it possible to track every Dane in a number of public registers. Previous studies have shown a high validity of Danish national registers $(21,22)$.

Sufficient confounder control. In theory, men exposed to occupational noise might have a different cardiovascular risk profile compared to unexposed men. In this study, only minor differences were observed with respect to lifestyle factors. The noise-exposed men were a little less sedentary during leisure time, whereas their accumulated lifetime smoking was slightly higher. With respect to clinical and metabolic factors, the long-term noiseexposed men had lower systolic and diastolic blood pressure, but higher fasting serum triglyceride levels. 
Also noise-exposed men more frequently belonged to low social classes. These established cardiovascular risk factors were included in the analyses. So, in this study, sufficient confounder control was obtained. Analyses stratified according to social class confirmed the overall results, further reducing the possibility that residual confounding may have been responsible for the lack of association between occupational noise exposure and the outcomes studied. The high number of end-points in this study makes it highly unlikely that a statistical type- 2 error may explain the results.

Interestingly, the results presented here are basically in agreement with the results from the only incidence study of occupational noise as a risk factor for IHD in the literature that appears to fulfill the aforementioned methodological challenges (4). The other cohort study nearly fulfilling the above criteria, that of Virkkunen et al (6), found an increased risk of coronary heart disease among men exposed to occupational noise. The major problem in that study was the crude classification of social class. Social class was only categorized as blue-collar worker or not. So residual confounding due to social class in that study cannot be ruled out, as suggested by the weaker association of noise exposure with risk of coronary heart disease among blue-collar workers. This may be the most likely explanation for the discrepancies between their results and those of our study and that of McNamee et al (4). Melamed et al (2) did not find an association between noise exposure and CVD mortality, but a statistically significant increased risk with respect to all-cause mortality. However, this may be due to the fact that only modest confounder control was applied, and there was no control for social class.

\section{Concluding remarks}

Cumulative occupational exposure to noise was strongly associated with hearing impairment. Although some misclassification of study participants' occupational noise exposure may exist, the present study renders no support for the hypothesis that occupational noise exposure is a risk factor for death from either IHD or allcauses in a long-term follow-up adjusting for established cardiovascular risk factors, including low social class.

\section{Acknowledgments}

The Copenhagen Male Study was supported by grants from King Christian X Foundation, the Danish Medical Research Council, the Danish Heart Foundation, and Else \& Mogens Wedell-Wedellsborg Foundation.

\section{References}

1. Nelson DI, Nelson RY, Concha-Barrientos M, Fingerhut M. The global burden of occupational noise-induced hearing loss. Am J Ind Med. 2005 December;48(6):446-58. http://dx.doi. org/10.1002/ajim.20223.

2. Melamed S, Kristal-Boneh E, Froom P. Industrial noise exposure and risk factors for cardiovascular disease: Findings from the CORDIS Study. Noise \& Health. 1999;4:49-56.

3. van Kempen EE, Kruize H, Boshuizen HC, Ameling $\mathrm{CB}$, Staatsen BA, de Hollander AE. The association between noise exposure and blood pressure and ischemic heart disease: a meta-analysis. Environ Health Perspect. 2002 March;110(3):307-17. http://dx.doi.org/10.1289/ ehp.02110307.

4. McNamee R, Burgess G, Dippnall WM, Cherry N. Occupational noise exposure and ischemic heart disease mortality. Occup Environ Med. 2006 December;63(12):813-9. http://dx.doi.org/10.1136/oem.2005.026245.

5. Davies HW, Teschke K, Kennedy SM, Hodgson MR, Hertzman C, Demers PA. Occupational exposure to noise and mortality from acute myocardial infarction. Epidemiology. 2005 January;16(1):25-32. http://dx.doi.org/10.1097/01. ede.0000147121.13399.bf.

6. Virkkunen H, Kauppinen T, Tenkanen L. Long-term effect of occupational noise on the risk of coronary heart disease. Scand J Work Environ Health. 2005 August;31(4):291-9.

7. Gyntelberg F. Physical fitness and coronary heart disease in male residents in Copenhagen aged 40-59. Dan Med Bull. 1973 January;20(1):1-4.

8. Gyntelberg F. One- and two-years incidence of myocardial infarction in copenhagen males aged 40-59. Dan Med Bull. 1975 February;22(2):81-4.

9. Svalastoga K. Prestige, class and mobility. Copenhagen: Munksgaard; 1959.

10. Suadicani P, Hein HO, Gyntelberg F. Serum validated tobacco use and social inequalities in risk of ischemic heart disease. Int J Epidemiol. 1994 April;23(2):293-300. http://dx.doi. org/10.1093/ije/23.2.293

11. Siedel J, Klose S, Ziegenhorn J, Wahlefeld AW. Improved reagent for the determination of serum cholesterol. J Clin Chem Biochem. 1981;19:838-9.

12. Stahler F, Gruber W, Stinshoff K, Roshlau P. Eine praxis gerechte enzymatische Cholesterin-Bestimmung [A practical enzymatic cholesterol determination]. Med Lab. 1977;30:29-37.

13. Burstein M, Scholnick HR, Morfin R. Rapid method for the isolation of lipoproteins from human serum by precipitation with polyanions. Lipid Res.1970;11:583-95.

14. Lopes-Virella MF. Cholesterol determination in high-density lipoproteins separated by three different methods. Clin Chem. 1977;23:882-4.

15. Stone NJ, Bilk, Rosenbaum S. Recent National Cholesterol Education Program Adult Treatment Panel III Update: Adjustments and Options. Am J Cardiol. 2005;96[suppl]:53E59E. http://dx.doi.org/10.1016/j.amjcard.2005.06.006. 
16. Friedewald WT, Levy RI, Fredrickson DS. Estimation of the concentration of low density lipoprotein in plasma, without use of the preparative ultracentrifuge. Clin Chem. 1972;18:499502.

17. Rose GA, Holland WW, Crowley EA. A sphygmomanometer for epidemiologists. Lancet. 1964;i:296-300. http://dx.doi. org/10.1016/S0140-6736(64)92408-0.

18. Cox DR. Regression models and life-tables. J R Stat Soc. 1972;34:187-220.

19. Jacobsen P, Hein HO, Suadicani P, Parving A, Gyntelberg F. Mixed solvent exposure and hearing impairment: an epidemiological study of 3284 men. The Copenhagen male study. Occup Med (Lond). 1993 Nov;43(4):180-4. http:// dx.doi.org/10.1093/occmed/43.4.180.
20. Parving A, Hein HO, Suadicani P, Ostri B, Gyntelberg F. Epidemiology of hearing disorders. Some factors affecting hearing. The Copenhagen Male Study. Scand Audiol. 1993;22(2):101-7. http://dx.doi. org/10.3109/01050399309046025.

21. Madsen M, Balling H, Eriksen LS. The validity of the diagnosis of acute myocardial infarction in 2 registers: the Heart Registry compared to the National Patient Registry. Ugeskr Laeger. 1990;152:308-14.

22. Mosbech J, Jørgensen J, Madsen M, Rostgaard K, Thornberg K, Poulsen TD. The Danish National Patient Register: evaluation of data quality. Ugeskr Læger. 1995;157:3741-5.

Received for publication: 23 May 2011 